\title{
The Relation of the Tensile \& Shear Stress of Schmid \& their Efficient Fracture Stress with Twins and Dislocations in TiAl Alloys
}

\author{
Run $\mathrm{Xu}^{1 *}$, Boyong Hur ${ }^{1}$, Sugun Lim ${ }^{1}$, Younwook Kim² \\ ${ }^{1}$ Gyeongsang National University, Metallurgical \& Materials Engineering Dept., Chinju 52828, Korea \\ ${ }^{2}$ Keimyung university, Materials Engineering Div., Daegu 42601, Korea
}

DOI: $10.36348 /$ sijcms.2022.v05i01.001

| Received: 05.12.2021 | Accepted: 09.01.2022 | Published: 12.01.2022

*Corresponding author: Run Xu

Gyeongsang National University, Metallurgical \& Materials Engineering Dept., Chinju 52828, Korea

\section{Abstract}

It is reasonable within the range from $45^{\circ}$ to $90^{\circ}$. It shows that it is maximum of the efficient resolved tensile and shear stress at $90^{\circ}$. The twin is the most active around $45^{\circ}$ roughly the most active range is 22.5 to $67.5^{\circ}$. Therefore we believe that the possibility of twin is relatively high. The effective stress according to $\sigma_{\text {eff }}^{2}=\sigma_{\mathrm{s}}{ }^{2}+\tau_{\mathrm{c}}{ }^{2}-2 \sigma_{\mathrm{s}} \tau_{\mathrm{c}} \cos \left(90^{\circ}+\phi\right)$ and the adapted effective stress $\sigma_{\text {eff }}^{2}=\sigma_{\mathrm{s}}{ }^{2}+\tau_{\mathrm{c}}{ }^{2}$ likely excludes a little deviation with $0 \sim 4 \mathrm{MPa}$ in $\mathrm{Ti}_{2} \mathrm{AlNb}$. The deviation is largest with $4 \mathrm{MPa}$ in $\phi$ of $42.5^{\circ}$ in $\mathrm{Ti}_{2} \mathrm{AlNb}$. The range rate is $0.5 \%$. According to efficient stress $\sigma_{\text {cal }}=\sqrt{\sigma^{2}+4 \tau^{2}}$ \& $\sigma_{\mathrm{ca} 2}=\sqrt{\sigma^{2}+3 \tau^{2}}$ twins and dislocation happen at $\lambda=45^{\circ}$ and $90^{\circ}$.

Keywords: Schmid stress and shear stress, efficient curve, compare relation, TiAl, efficiency deviation.

Copyright (C) 2022 The Author(s): This is an open-access article distributed under the terms of the Creative Commons Attribution 4.0 International License (CC BY-NC 4.0) which permits unrestricted use, distribution, and reproduction in any medium for non-commercial use provided the original author and source are credited.

\section{INTRODUCTION}

The Schmid factor is the one factor to control CRSS in tensile. The CRSS is one to cause shear stress and strain in slope. CRSS and RTS are two important respects to evaluate material tensile properties. So we calculate the two parameters has significant role. Meantime we gain the corresponding effective stress to observe the synthetic features. We know the twins and dislocations are two defects in tensile experiment. But we cannot know the detailed information to cause by now. In general the twins caused by shear and dislocations caused by tensile and shear too. We want to know the information resulting the fracture factors through this paper. As we know the dislocations are caused by shear and tensile stress both with the slip planes like (111) and (110\} in materials [1-6]. They are moved through these group planes by shear stress or a certain plane by tensile stress. Twins like dislocations but their formation is mainly shear stress with symmetric plane like (1012\}, (111) etc. The Schmid factor causes dislocation happens to slip easily if they are small enough. If they are large the dislocation is hard to happen with certain value. CRSS makes important role in order to observe the dislocation happen easily or hard to a certain materials. So we judge the CRSS value to observe the certain value to cause dislocation or twins. In Ti-Al alloys the small value to calculate to simulate the dislocation happening model to expose the dislocation \& twins happen condition in this study.

Deviation is used to evaluate the calculating results and effective stress between RTS and CRSS in this study. A little deviation is found effective stress between RTS and CRSS. Good deviation is gained here regarding to complete data. 


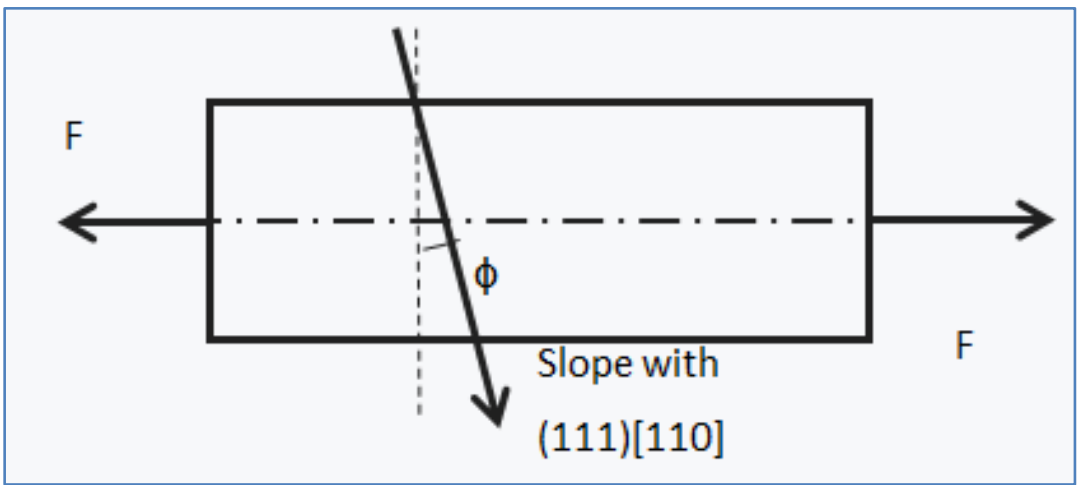

Fig-1: The Schmid factor schemetic in the tensile

\section{Calculation results}

As shown in Figure 1 the [110] direction is in the slope plane. The schmid factor is based on this plane. The Schmid factor is

$\operatorname{Cos} \lambda \cos \phi(1)$

And CRSS (critical resolved shear stress) is below $\tau_{\mathrm{c}}=\sigma_{\mathrm{s}} \cos \lambda \cos \phi$ (2)

RTS (resolved tensile stress) is $\sigma=\sigma_{s} \sin \lambda \cos \phi(3)$

Here $\sigma_{\mathrm{s}}$ is yield stress. $\lambda$ is $90^{\circ}-\phi . \phi$ is angle between slip plane and cross section..

The effective stress is according to $\sigma_{\text {eff }}^{2}=\sigma_{\mathrm{s}}{ }^{2}+\tau_{\mathrm{c}}{ }^{2}-2 \sigma_{\mathrm{s}} \tau_{\mathrm{c}} \cos \left(90^{\circ}+\phi\right)$

The adapted effective stress is $\sigma_{\text {eff }}^{2}=\sigma_{\mathrm{s}}^{2}+\tau_{\mathrm{c}}^{2}(5)$

The deviation between above two stresses is calculated with variance and standard deviation. Produced by twin shear stress and shear stress by the axial force into a certain Angle theta, the maximum at $45^{\circ}$ formation, so its most easily formed in this Angle, the number is also the most. By analyzing the distribution of shear stress and found it in $45^{\circ}+/-22.5$ ${ }^{\circ}$ distribution at most. It has the closest relationship to the bevel Angle of the cross section. Transposition is produced by tensile stress parallel to the external force. By tensile stress component is decomposed in inclined section of the tensile stress also can produce dislocation, so its distribution in $0^{\circ} 22.5 \mathrm{~mm}$ at most. It's closest to being reorganized. Therefore, we hypothesized that the twin crystals mainly acted on the cross section and the transposition mainly acted on the axial section.

As shown in Figure 2, in ti-al alloy, CRSS and Schmid factors are related to being rearranged as normal increasing functions. Decomposition of critical shear stress value maximum around $45^{\circ}$, the decomposition of shear stress parallel to the cross section, so we estimate that the largest reduction of area under shear stress. Figure 2 also shows that RTS and Schmid factors are related to being rearranged as a convex delta function. But its growth is larger than CRSS shows that the tensile stress in the cross section is around $45^{\circ}$, the largest tensile stress and vertical section, so the tensile stress influence inversion. The effective stress curve is shown in figure. It is calculated with $\sigma_{e f}=\sqrt{\sigma^{2}+\tau^{2}}$ and find the size is gradually increasing with the $\lambda$ increasing. It can be seen from figure 3 that in Ti2AlNb alloy, the increase of CRSS and RTS is larger than that of capital, indicating that the critical decomposition stress of twin crystals and translocation is larger. The effective stress is calculated with $\sigma_{e f}=\sqrt{\sigma^{2}+\tau^{2}}$ and find the size is gradually increasing with the $\lambda$ increasing too. If the fracture cross is inclined the shear stress will be happened. The twins will be happened too. However, the Schmid factor in $\mathrm{Ti} 2 \mathrm{AlNb}$ is as large as that in capital, indicating that it is difficult for them to have cleavage faults. In TiAl alloy $1 / 2$ Max shear stress is equal to the largest when the $45^{\circ}$, the twinning and dislocation along the surface of super inversion (112) and sliding to the maximum. At $90^{\circ}$ the Max tensile stress in complete transposition $1 / 2$ (110) twinning and dislocation are produced, however, the twin dislocation. Prismatic translocation $<1120>(1010)$ exists outside Ti2AlNb alloy $<1120>(0001)$.A transposition exists with double $1 / 6 \mathrm{a}<1120>$ overtransposition. Decomposition of tensile stress in these alloys section form the maximum value from 0 to $90^{\circ}$, formed in the dense surface inversion and a small amount of twin began to form joint action of plastic deformation. Schmid factor and a close relationship with CRSS, at $45^{\circ}$ shear stress prompted twin formation system and formation of a certain number of transpositions. Because the tensile stress is half of the shear stress, there is no large amount of transposition in the tensile stress plane. Twinning is twice as much as transposition. Based on analysis of dislocation is active within the range of $45^{\circ}$ to $90^{\circ}$, it shows that it is with the tensile stress at $90^{\circ}$ Angle. And twinning is most active around $45^{\circ}$ roughly the most active within the range of $22.5^{\circ}$ and $67.5^{\circ}$. Based on this, we believe that the twin crystal produces crosssection shrinkage and translocation produces elongation evidence. 


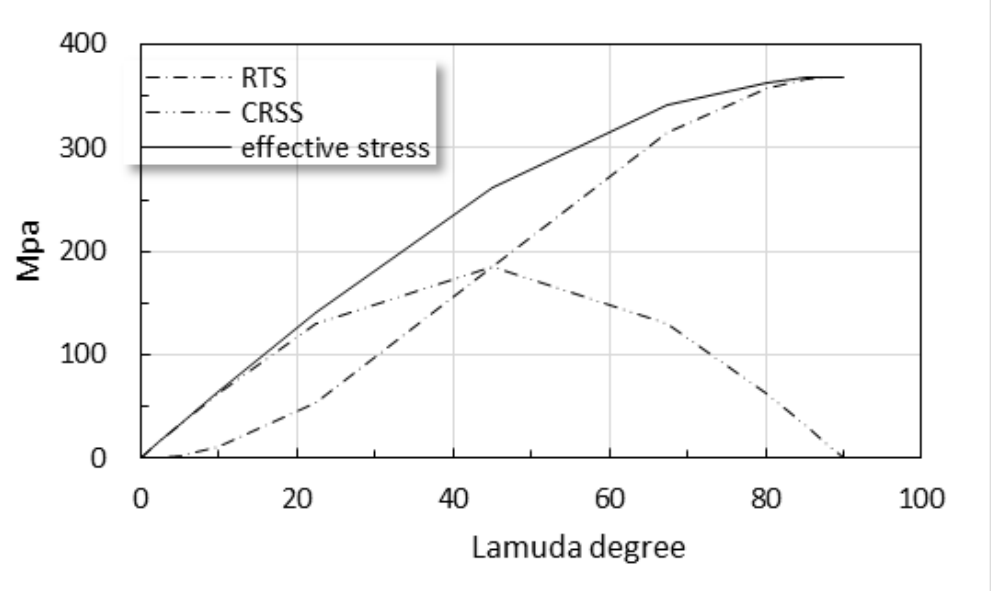

Fig-2: The relations of $\lambda$, RTS\& CRSS in TiAl

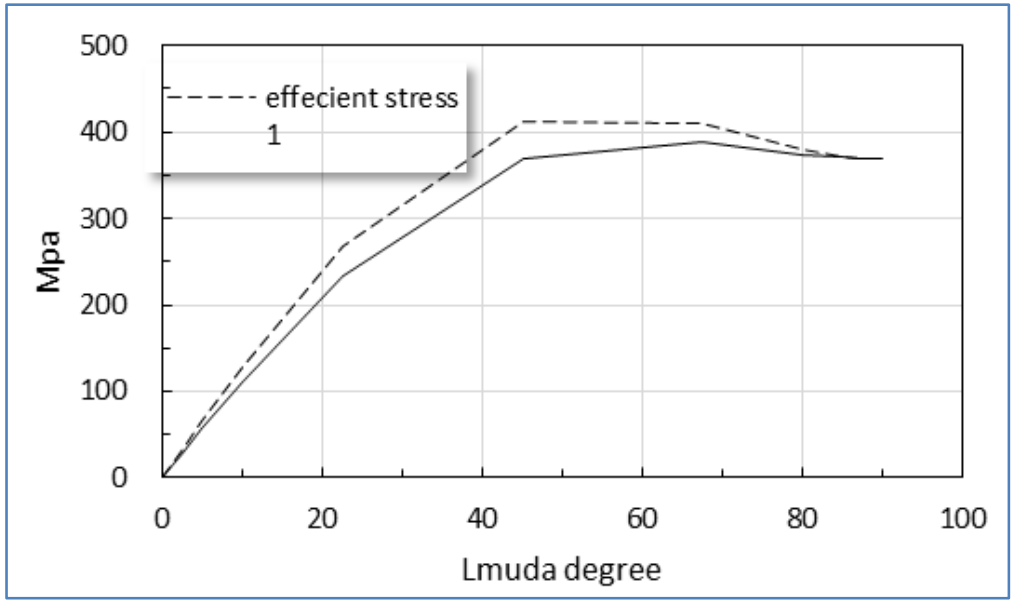

Fig-3: The relations of $\phi \&$ effective stress and $\Delta$ eff stress in TiAl

The curves calculated according to the equivalent stress are shown in the Figure 2. Curve 1 was the result calculated with the former while curve 2 was the result calculated with the latter. The equivalent stress is slightly higher. So we know that the equivalent stress increases as lambda increases. The maximum value occurs between 45 and 90.This indicates that twin crystals are produced at $45^{\circ}$ and translocation occurs at $90^{\circ}$.The failure surface is double crystal failure at $0 \sim 60^{\circ}$, or dislocation failure at $90^{\circ}$. At $45^{\circ}$ to $90^{\circ}$ these inclined planes produce maximum equivalent stress, i.e., double crystal and few transpositions are formed.

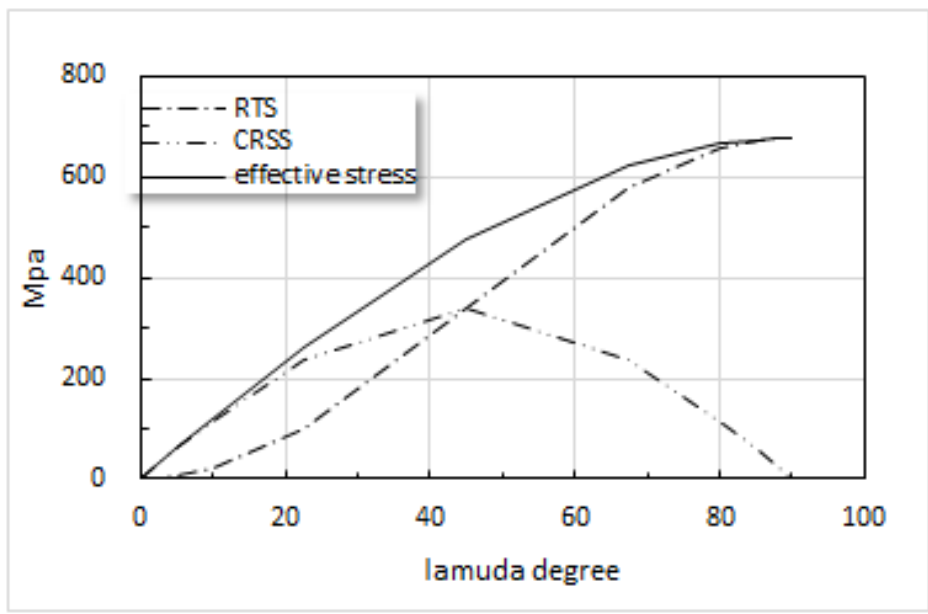

Fig-4: The relations of $\lambda$ and CRTS \& CRSS in $\mathrm{Ti}_{2} \mathrm{AINb}$ 


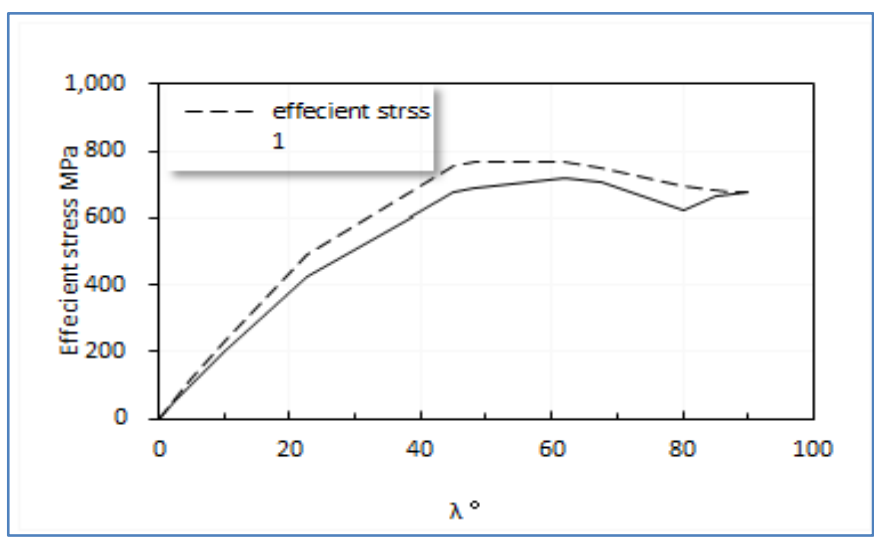

Fig-5: The relations of $\phi \&$ effective stress and $\Delta$ eff stress in $\mathrm{Ti}_{2} \mathrm{AlNb}$

Table-1: The values and deviations of yeild stress $\&$ fracture stress in Ti-Al

\begin{tabular}{|l|l|l|l|l|l|}
\hline Strength & \multirow{2}{*}{$\mathbf{S}_{\mathbf{s}} \mathbf{M P a}$} & \multirow{2}{*}{$\mathbf{s}_{\mathbf{b}} \mathbf{M P a}$} & $\mathbf{G}$ & $\mathbf{E}$ & CRSS(RTS) \\
\cline { 1 - 4 } Sorts & & & GPa & GPa & $45^{\circ}$ \\
\hline$\gamma$ & 369 & 454 & 59 & 150 & 184 \\
\hline literature-TiAl & 367 & 526 & 64 & 168 & 183 \\
\hline standard deviation $\%$ & 0.27 & 7.35 & 4.07 & 5.66 & 0.27 \\
\hline$\alpha_{2}$ & 676 & 845 & 45 & 111 & 338 \\
\hline literature-Ti ${ }_{2} \mathrm{AlNb}$ & 591 & 847 & 42 & 123 & 296 \\
\hline standard deviation $\%$ & 6.71 & 0.12 & 3.45 & 5.13 & 6.62 \\
\hline
\end{tabular}

The curves of equivalent stress and calculation according to the third and fourth strength theories are shown in the figure. Curve 1 is the result calculated by the former in $\mathrm{Ti}_{2} \mathrm{Al}$, while curve 2 is the result calculated by the latter. The equivalent stress is slightly higher. So we know that the equivalent stress increases as lambda increases. The maximum value occurs between 45 and 90.This indicates that the maximum equivalent stress, i.e., double crystal and few transpositions will be generated on these inclined planes.
Table-2: The values of $\Delta$ effective stress $\&$ range deviation in Ti-Al

\begin{tabular}{|l|l|l|}
\hline types & items & values \\
\hline & effective stress & 375 \\
\hline TiAl alloy & Deffective stress & 2.2 \\
\hline & Range rate $\%$ & 0.58 \\
\hline & effective stress & 680 \\
\hline $\mathrm{Ti}_{2} \mathrm{AlNb}$ alloy & $\Delta$ effective stress & 3.8 \\
\hline & Range rate $\%$ & 0.55 \\
\hline
\end{tabular}

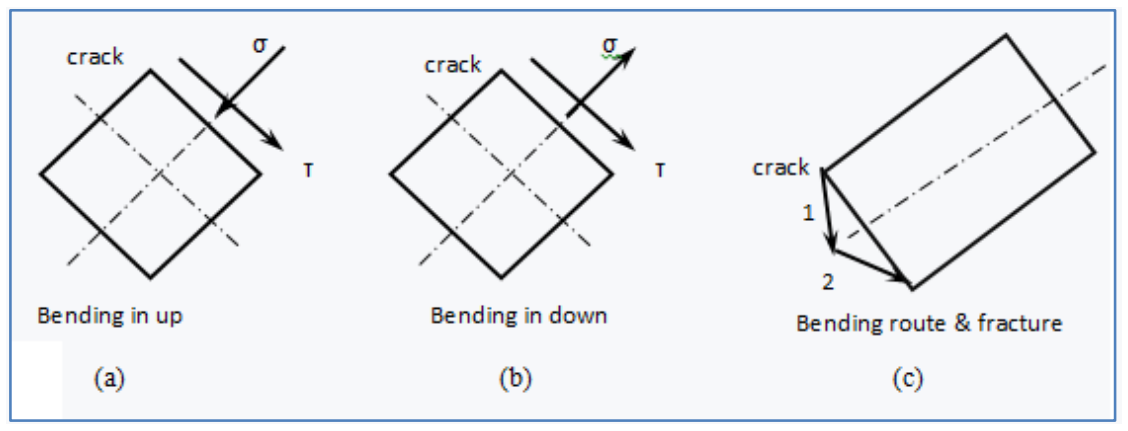

Fig-6: The crack happening in bend test

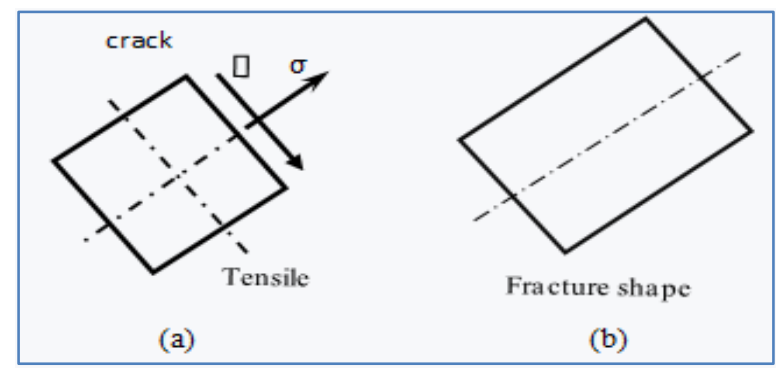

Fig-7: The crack happening in tensile test 
TEM diagram showed that [111] formed an Angle of about $60^{\circ}$ with gamma. Gamma lattice structure shows that theta $=55^{\circ}$ is close to $60^{\circ}$, so it can be considered that the direction of layered tissues is gamma //[2110] alpha 2. Alpha 2 is vertically distributed with the direction of layered tissues, which is not conducive to strength. It should be $45^{\circ}$ from the lamellar direction to be the best direction. It is good for both strength and ductility, so about $45^{\circ}$ is the ideal direction.

The vertical direction [101] in this direction is the Burgers vector B. Super inversion $1 / 2[110]=1 / 6 * 2[111]+1 / 6[11 \overline{2}]+\mathrm{SESF}$ as seen in Figure 8.

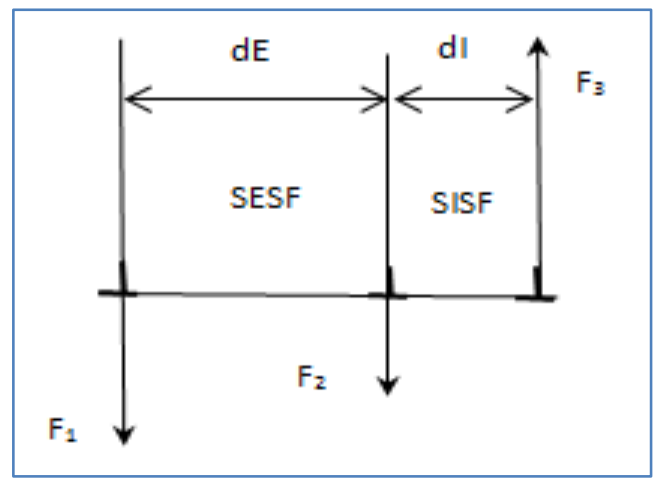

Fig-8: The dislocation of a [112]/2 edge super dislocation

Here SE/ISF is the super lattice extrinsic/intrinsic stacking fault energy. $\mathrm{F} 1 \sim \mathrm{F} 3$ is the force of dislocation.. Here $F_{2}=F_{3}-F_{1}$ ie. $F_{3}=F_{1}+F_{2} . F_{3}$ is the $1 / 2[110], F_{1}$ is the $1 / 3[111]$ and $F_{2}$ is the $1 / 6[11 \overline{2}]$ in Figure 7 . A Frank partial of $\mathrm{Bf}$ is [111]/3 and Shockley partial of Bs is [112]/6 bound loop. In terms of the calculation of anisotropic elastic line energy dissociation is feasible as the Shockley partial which is the edge or a mixed character. The force may be through above curves to get in a certain plane in Figure 2 and 3. Moreover the formula has $\mathrm{dI}^{*} \mathrm{~F}_{3}=\mathrm{dE}^{*} \mathrm{~F}_{1}$ which means we can get the ratio of forces according to distances.

In Figure 6 in bending tests, as in the threepoint bend test, the fracture mechanism is based on shear stress, which starts at the upper end of the specimen and ends at the lower end after passing through the middle neutral plane. Because the largest number is at the upper end, the neutral side is 0.And sigma is the biggest upper here. So it's the fracture mechanism. In tensile tests in Figure 7, however, the cracks are evenly distributed in the diagonal section. They begin in the middle and continue up and down the section. And sigma usually starts in the middle and finishes at the two bottoms. It belongs to the inter crystalline fracture and no fracture mechanism. And sigma belongs to the realm fault and the ductile fault. It is also called "honeycomb" fault. If you want to improve the ductility; you can improve the sigma influence.

\section{CONCLUSIONS}

1. Based on the efficiency resolved curve of shear stress and tensile stress it is reasonable within the range from $45^{\circ}$ to $90^{\circ}$. It shows that it is maximum of the efficient resolved tensile and shear stress at $90^{\circ}$.

2. The twin is the most active around $45^{\circ}$ roughly the most active range is 22.5 to $67.5^{\circ}$. Therefore we believe that the possibility of twin is relatively high. The effective stress according to $\sigma_{\text {eff }}^{2}=\sigma_{\mathrm{s}}{ }^{2}+\tau_{\mathrm{c}}{ }^{2}-2 \sigma_{\mathrm{s}} \tau_{\mathrm{c}} \cos \left(90^{\circ}+\phi\right)$ and the adapted effective stress $\sigma_{\text {eff }}^{2}=\sigma_{\mathrm{s}}{ }^{2}+\tau_{\mathrm{c}}{ }^{2}$ likely excludes a little deviation with $0 \sim 4 \mathrm{MPa}$ in $\mathrm{Ti}_{2} \mathrm{AlNb}$. The deviation is largest with $4 \mathrm{MPa}$ in $\phi$ of $42.5^{\circ}$ in $\mathrm{Ti}_{2} \mathrm{AlNb}$. The range rate is $0.5 \%$.

3. According to efficient stress $\sigma_{\text {cal }}=\sqrt{\sigma^{2}+4 \tau^{2}}$ \& $\sigma_{\mathrm{ca} 2}=\sqrt{\sigma^{2}+3 \tau^{2}}$ twins and dislocation happen at $\lambda=45^{\circ}$ and $90^{\circ}$.

\section{REFERENCES}

1. Run, X., \& Sugun, L. (2019). Analysis on deviation and necking formula between the elongaion \& reduction I [J], Materials Science, 9(1), 62-68.

2. Run, X., \& Sugun, L. (2018). Analysis on deviation and necking formula between the elongaion \& reduction in tensile test $[\mathrm{J}]$. Materials Science, 8(11), 1027-1031.

3. Shimada, Y., Inui, H., \& Yamaguchi, M. (1994). Proc of the $5^{\text {th }}$ symp om High-performance materils for severe environments, 23

4. Shao, G., Tsakiropoulos, P., \& Miodownil, A. P. (1997). Materials Science and Technology, 13; 97

5. Johnson, D. R., Inui, H., \& Yamaguchi, M. (1996). Acta Metall., 44; 2523

6. Nishiyama, Y., Miyashita, T., Isobe, S., \& Noda, T. (1990). High Temperature Auminides and Intermetallics, S. H. Whang. (Eds), TMS, 557. 